\title{
VASCULAR SYMPTOMS AND DIGITAL PLETHYSMOGRAPHY ABNORMALITIES IN THE FEET OF WORKERS WITH HAVS
}

\author{
Ron House, ${ }^{1 *}$ Aaron Thompson, ${ }^{1}$ Tammy Eger, ${ }^{2}$ Kristine Krajnak, ${ }^{3}$ Depeng Jiang ${ }^{1}$ \\ 1. University of Toronto and St. Michael's Hospital, Toronto, Ontario, Canada \\ 2. Laurentian University, Sudbury, Ontario, Canada \\ 3. Health Effects Laboratory Division, National Institute for Occupational Safety \\ and Health, Morgantown, WV, USA.
}

\section{Introduction}

The vascular component of Hand-Arm Vibration Syndrome (HAVS), often referred to as vibration white finger (VWF) has been known for many years. There is also evidence to suggest that HAVS may be associated with cold-induced vasospastic abnormalities in the feet. ${ }^{2}$ This is an important issue in terms of prevention and compensation, especially in light of the large size of the exposed population. This study was carried out to determine the prevalence and risk factors of cold-induced vasospastic changes in the feet in a group of construction workers who were assessed for HAVS at the Occupational Health Clinic, St. Michael’s Hospital, Toronto.

\section{Methods}

The subjects in this study were 191 male construction workers. Their occupational histories indicated that they were exposed to hand-arm vibration without significant vibration exposure directly to the feet. Digital photocell plethysmography was carried out in the hands and feet of all subjects and consisted of baseline measurement of blood flow in the fingers and toes followed by measurement after cold water immersion $\left(10^{\circ} \mathrm{C}\right.$ for 2 minutes). The scoring was based on the amplitude of the waveforms and the change in amplitude after cold water immersion as follows: 0: all waveforms normal; 1: mild changes; 2: mixture of mild and moderate changes; 3 : moderate changes; 4 : mixture of moderate and severe changes; 5: severe changes. Binary logistic regression was carried out to examine the relationship between the digital plethysmography findings in the feet and various predictor variables. In this analysis, the digital plethysmography scores in the feet were dichotomized into severe cold induced changes in waveforms (stages 4 or 5) in both feet versus not having any severe waveform changes in both feet. The predictor variables examined included a history of cold intolerance in the feet, years worked in construction, type of construction trade, the average Stockholm vascular scores in both hands combined and the average digital plethysmography scores in both hands combined. Interaction terms were also examined. In all of the models an initial saturated model was fitted followed by backwards stepwise elimination to produce the final model.

\section{Results}

The subjects had a median age of 57 years (range: 28-75) and a median number of years worked in construction of 36 (range: 4-52). One hundred and sixty- nine (88.5\%) of 
the workers reported cold intolerance in their feet. The digital plethysmography results indicated that most workers had some degree of objective cold-induced vasospasm in the hands and feet. Only10 workers had normal (stage 0) results in the right and left hands and only one worker had normal results in the right and left feet. Severe cold-induced changes in waveforms in at least one digit (i.e. stages 4 or 5) were found in the right foot in 59 (30.9 \%) subjects, in the left foot in 62 (32.5\%) subjects, and in both feet in 49 (25.7\%) subjects. The results of the binary logistic regression indicated that in the saturated model the only statistically significant predictor of severe (stage 4 or 5) vascular abnormalities in both feet was the average digital plethysmography score in the hands (odds ratio: 1.69; 95\% CI: 1.18 - 2.42). No interaction terms were found to be statistically significant. After backwards stepwise elimination the average digital plethysmography score in the hands continued to be the only statistically significant predictor of objective vascular abnormalities in the feet and the odds ratio (95\% CI) increased to 1.87 (1.35-2.59).

\section{Discussion}

This study has shown a high prevalence of vascular abnormalities in the feet of workers with HAVS. A total of $88.5 \%$ of study participants reported cold intolerance in their feet. The digital plethysmography indicated that $99.5 \%$ of subjects had objective evidence of at least mild cold induced vasospastic changes in each foot and the percentage with at least one digit showing severe changes was 30.9\% in the right foot and $32.5 \%$ in the left foot. The logistic regression analysis indicated that the key predictor of severe vascular changes in the feet was the extent of digital plethysmography changes in the hands.

In a recent literature review Schweigert ${ }^{2}$ indicated that there was evidence to suggest that vascular effects in the lower extremities may be associated with VWF from hand-arm vibration exposure. This is consistent with the results of our study. Hand-arm vibration exposure and HAVS may be associated with both local and central sympathetic stimulation. ${ }^{3}$ As well Bovenzi et al. ${ }^{1}$ have recently shown that salivary concentrations of the potent vasoconstrictor endothelin ET (1-21) are increased in forestry workers with VWF in comparison to controls which suggests another mechanism for vasospasm in the feet in workers with HAVS.

Our study findings are relevant to understanding the spectrum of health effects associated with hand-arm vibration. Future work could focus on the functional significance of these vascular findings in the feet and their prevention.

\section{References}

1. Bovenzi, M., D’Agostin, F., Rui F, Ambrosi, L., Zefferino, R. (2008). Salivary endothelin and vascular disorders in vibration-exposed workers. Scand J Work Environ Health. 34,133-141.

2. Schweigert, M. (2002). The relationship between hand-arm vibration and lower extremity clinical manifestations: a review of the literature. Int Arch Occup Environ Health. 75,179-185.

3. Stoyneva, Z., Lyapina, M., Tzvetkov, D., Vodenicharov, E.(2003). Current pathophysiological views on vibration-induced Raynaud’s phenomenon. Cardiovas Res. 57,615-624. 